Chapter 2

\title{
Cardiorespiratory Complications During Moderate and Deep Sedation for Gastrointestinal Endoscopic Procedures
}

\author{
Somchai Amornyotin \\ Additional information is available at the end of the chapter \\ http://dx.doi.org/10.5772/52737
}

\section{Introduction}

Gastrointestinal endoscopic (GIE) procedures are now performed routinely because of their minimal invasiveness and their diagnostic and therapeutic capabilities. These procedures have been shown to cause various effects on cardiorespiratory systems, which can increase the risks of the procedure in patients with underlying cardiorespiratory diseases [1]. Additionally, the complications attributed to moderate and deep sedation levels are more often associated with cardiovascular and respiratory systems. Most predictors of cardiorespiratory-related complications are patient-centered factors and do not vary significantly from procedure to procedure although the procedure is complex [2].

The exact incidence of cardiorespiratory complications associated with GIE procedure is not precisely known, but probably is quite low. Risk factors for cardiorespiratory complications are age $>60$ years, high American Society of Anesthesiologists (ASA) physical status, the use of supplemental oxygen, inpatient status, and the involvement of a trainee in the procedure $[3,4]$. Sedation-related complications during GIE procedures are commonly transient and mild degree. The risk for these complications while providing any level of sedation or general anesthesia is greatest when caring for the patients already medically compromised. The significant unwanted complications can generally be prevented by careful preprocedure assessment and preparation, appropriate monitoring and support as well as postprocedure management.

Before undertaking any GIE procedure, endoscopists should be obtained the informed consent from the patient, are familiar with the latest guidelines on sedation, aware of any medical, surgical and drug history elicited in the pre-admission process as well as the risk factors should 
be identified in both out-patients and in-patients. Additionally, the physicians must be prepared to manage these complications. Safety and monitoring should be part of a quality assurance program for endoscopy units. This article will review the cardiovascular and respiratory complications during moderate and deep sedation for gastrointestinal endoscopic procedures and also address their appropriate management.

\section{Cardiovascular consideration}

The autonomic nervous system (ANS) plays an important role in maintaining normal hemodynamics and an adequate coronary blood flow. The sympathetic nervous system regulates the heart rate and rhythm, increases the excitability of myocardium. The parasympathetic nervous system regulates the heart rate and rhythm, which when stimulated can lead to sinus bradycardia [1]. The cardiorespiratory complications account for about $50 \%$ of the potentially serious morbidity and about $50 \%$ of all the procedure-related deaths associated with GIE procedure. In many cases these complications are a direct or indirect consequence of elderly or risk patients being given unnecessarily high doses of sedative and analgesic drugs [5].

\subsection{Hypotension}

A significant decline in blood pressure from baseline should alert the clinicians. Hypotension is defined as the systolic blood pressure lest than $90 \mathrm{mmHg}$ is due to a fall in either cardiac output or total peripheral resistance lowering the patient's mean arterial pressure.

Episodes of hypotension in clinical practice are most commonly associated with vasovagal events and are generally transient. However, they may become prolonged in the presence of central nervous system depressants [6]. Blood pressure is a reflection of cardiac output and total peripheral resistance and a fall in either or both will lower the patient's mean arterial pressure. In general, a systolic blood pressure of $90 \mathrm{mmHg}$ should sustain mean arterial blood pressure sufficiently to perfuse tissues in the recumbent patient. Blood pressure lower than this combined with evidence of inadequate perfusion requires intervention.

The evaluation of tissue perfusion is the most significant component of cardiovascular assessment. Hypotension encountered during sedation is usually attributed to either vasovagal episodes or the use of sedative and anesthetic agents that depress sympathetic outflow to the cardiovascular system. Benzodiazepines such as midazolam and diazepam, have a mild vasodilator effect and usually produce a slight fall in arterial blood pressure even in normal sedative doses. The combination use of a benzodiazepine and an opioid can profoundly drop blood pressure. Propofol has been shown to be safe and effective for sedation during ERCP, endoscopic ultrasonography (EUS) and small bowel enteroscopy, because these procedures require more time and patient cooperation [7-11].

The cardiovascular effects of propofol include decreases in cardiac output, systemic vascular resistance, and arterial pressure. A fall in heart rate and/or cardiac stroke volume will also lower blood pressure. Additionally, more profound falls in blood pressure is occurred in a 
hypovolemic patient. Propofol also has been proven to reduce postprocedural hypoxemic events, which may be of significance in critically ill elderly patients [12] and sick pediatric patients [13].

Prevention of this complication is to be relevant medical and drug history before the procedure, particular detail required regarding current antihypertensive, anti-anginal and anti-arrhythmic therapy and the use of systemic corticosteroids. Additionally, blood pressure and heart rate should be recorded before, during and after endoscopic procedure.

\subsection{Hypertension}

Blood pressure continuously fluctuates due to the cyclic nature of the pumping action of the heart. The highest pressure occurs during ventricular contraction. The lowest pressure occurs during ventricular relaxation [14]. Generally, hypertension is defined as the systolic blood press is greater than $160 \mathrm{mmHg}$. Sudden elevations of systolic blood pressure $\geq 180 \mathrm{mmHg}$ or diastolic blood pressure $\geq 110 \mathrm{mmHg}$ are generally regarded as an acute hypertensive episode [5]. The causes of hypertension are the background systemic hypertension, anxiety or pain, and a reflex pressor response from intubation of the esophagus. Generally, asymptomatic patients and the patients without acute end-organ symptoms should not receive antihypertensive agents in the endoscopy unit.

\subsection{Cardiac arrhythmias}

Autonomic control of heart rate will respond to demands placed on the patient and may be initiated via several baroreceptor-mediated reflexes [12]. Electrocardiogram (ECG) is also a useful monitor for heart rate and a better assessment of heart rhythm. Continuous ECG monitoring is recommended for high risk patient with relevant cardiac history. Cardiac arrhythmias are frequently observed during GIE procedures. Fortunately, most of them are not clinically significant.

In the healthy patients, a heart rate up to 120 beats/min will usually allow adequate filling. Sinus tachycardia can be caused by patient's anxiety or related to pain, compensatory mechanism in patients who are hypotensive as a result of either dehydration or blood loss, and following intravenous anticholinergic drugs such as buscopan.

Heart rate $<50$ beats/min in healthy patients may allow for more time in diastole, but ventricular filling becomes maximized [14]. Sinus bradycardia is most frequently seen in the patients who are taking beta blockers. It can also be induced by vagal stimulation, which occurs at the time of intubation of the esophagus or the stretching of the sigmoid mesentery during colonoscopy or flexible sigmoidoscopy.

\subsection{Myocardial ischemia/infarction}

Myocardial infarction occurs either during or in the few days after endoscopic procedures with or without sedation. A proportion of these are undoubtedly causally related to the endoscopic procedure. The causes of angina or myocardial Infarction are two factors; increased myocardial oxygen demand and reduced myocardial perfusion [6]. 
Increased myocardial oxygen demand is due to an increase in the mean arterial blood pressure and heart rate. This can cause angina in the patients with ischemic heart disease or occult symptomless myocardial ischemia. Additionally, marked hypertension and/or tachycardia increase myocardial oxygen consumption. In the other way, hypotension and/or bradycardia reduce myocardial perfusion. Stress-induced myocardial ischemia can occur even in the patients with or without clinically significant coronary disease [15]. This myocardial ischemia is related to the activation of the sympathetic nervous system, resulting in hemodynamic changes causing an increase in cardiac demand.

Prevention or minimization of myocardial ischemia/infarction during GIE procedure

1. Pre-oxygenation in risk patients and give continuous supplemental oxygen

2. Give patients on their normal anti-hypertensive and/or anti-anginal therapy right up to the time of the endoscopy

3. Angina developing during an endoscopy is usually best managed by giving sublingual nitroglycerine, oxygen supplementation and discontinuing the examination

4. If angina or myocardial infarction is suspected during or following an endoscopy, arrange an electrocardiogram to exclude an myocardial infarction

\section{Respiratory considerations}

Airway management is the most important aspect of patient care and examination of the patient's airway is an essential component of the preoperative assessment. Mallampati class correlates with increased difficulty in airway management. High oxygen concentration is indicated for patients who are spontaneously breathing, regardless of their level of consciousness during medical urgencies and emergencies. The equipment required to provide supplemental oxygen includes a $100 \%$ oxygen source, a regulator, tubing, and either a nasal cannula or mask. Every office should be equipped with a portable E-cylinder of oxygen.

\subsection{Respiratory depression}

Higher dose of benzodiazepine and/or opioid is also the greater the percentage benzodiazepine and/or opioid receptor occupancy in the central nervous system the greater is the degree of depression of consciousness. Intravenous benzodiazepines such as midazolam and diazepam can cause respiratory depression. Intravenous opioids such as pethidine and fentanyl occupy opioid receptor sites within the brain and brainstem and can similarly cause respiratory depression [6]. Drug induced hypoventilation may cause both hypoxemia and $\mathrm{CO}_{2}$ retention.

Pulse oximetry is a very useful indicator of oxygenation but not ventilation. However when supplemental oxygen is used, the fall in $\mathrm{SpO}_{2}$ may be significantly delayed for between 30-90 seconds. So that continuous capnography monitoring is recommended in the patients being sedated with propofol [16]. As for over-sedation, loss of verbal contact due to reduced conscious level may be the first sign of impending respiratory depression. Reduction in 
$\mathrm{SpO}_{2}$ on pulse oximetry is a good indicator but it can be a late sign of respiratory depression. Increased $\mathrm{PaCO}_{2}$ is the most sensitive early warning of respiratory depression [17].

Management of oversedation is to stimulate the patient to wake up and take deep breaths both verbally and/or light shaking. If the patients are not responding then benzodiazepine antagonist such as flumazenil and/or opioid antagonist such as naloxone may be required. The airway may need to be protected with chin lift, jaw thrust, and, if necessary, airway or laryngeal mask [6].

\subsection{Airway obstruction}

Although, obstruction may result in hypoventilation and hypoxia. Airway obstruction must be distinguished from respiratory depression. Hypoxia is common in patients undergoing upper GIE procedure with or without sedation. Sedation significantly increases the incidence of desaturation and hypoxia. Supplementary nasal oxygen at 3 litres/min in sedated patients abolishes desaturation and hypoxia. Upper airway obstruction may be attributed to anatomical structures or foreign body [18]. Independent predictors of airway modifications include male sex, American Society of Anesthesiologists class of III or higher, and increased body mass index [19].

Laryngospasm is a reflex closure or spasm of the glottic muscles including the false and true vocal cords. It is more likely during deep sedation. Laryngospasm occurs more frequently in adults who are smokers. Bronchospasm is a lower airway obstruction due to contraction or spasm of the bronchial smooth muscle. It may be a result of an anaphylactoid reaction or a consequence of a hyper-reactive airway in the asthmatic patients [18]. Management of laryngospasm and bronchospasm depends on the severity and the causes of them.

\subsection{Hypoxia}

Hypoxia may be a consequence of respiratory depression or airway obstruction. The incidence of hypoxia is up to $1.5 \%$ to $70 \%$, which make it the most common cardiorespiratory adverse event during the endoscopy [20]. Hypoxemia can lead to many complications, depending on the severity of hypoxemic attack. The use of supplemental oxygen during GIE procedure is routinely used by many endoscopists. However, oxygen supplementation will delay the detection of apnea and hypoxia [4]. Additionally, in patients given supplemental oxygen, saturation may be maintained in the progression of hypercapnia.

Multivariable logistic regressions revealed that independent risk factors for hypoxemia include high body mass index, hypertension, diabetes, gastrointestinal diseases, heart diseases and the procedures that combined esophagogastroduodenoscopy (EGD) and colonoscopy [21]. Hypoxemia occurs typically within 5 min of medication administration or endoscope intubation and only one third of all apnea and abnormal ventilation events eventually lead to hypoxemia [20].

\subsection{Pulmonary aspiration}

Aspiration of gastric contents into the lungs during GIE procedure is relatively common. It may cause pneumonia and may result in death. Risk factors for aspiration are the elderly 
patients, over-sedated patients, the patients with gastrointestinal bleeding, gastric stasis, gastric outlet obstruction, patients with hepatic encephalopathy, and the patients who have full stomach. Aspiration can also occur when a local anesthetic spray is used in combination with intravenous sedation [6].

Aspiration may be suspected when a patient starts coughing violently either during or soon after an endoscopic procedure and cyanosis may occur. Although the higher incidence of pulmonary aspiration because of the better sensitivity of $2-\left[{ }^{18} \mathrm{~F}\right]$ fluoro-2-deoxy-D-glucose positron tomography. However, the low incidence of clinical events needed intervention may still reflect the safety of sedation used for gastrointestinal endoscopy [22]. Treatments of pulmonary aspiration include suction of fluids from oral cavity and throat, increasing the rate of supplemental oxygen, encouraging the patient to cough, chest film, antibiotics and physiotherapy.

\section{Patients requiring anesthesiologists support for GIE procedures}

Generally, GIE procedures can be performed by using topical anesthesia, intravenous sedation and general anesthesia [23-25]. The topical anesthesia and intravenous sedation techniques can be effectively done by non-anesthetic personnel. However, non-anesthetic personnel should be sedated the patients only in mild and moderate (conscious) sedation levels [26].

Elective cases - Indications include:

1. Hypotension (systolic blood pressure $<90 \mathrm{mmHg}$ ) due to a fall in cardiac output or total peripheral resistance

2. Patients with severe cardiac and/or pulmonary abnormalities

3. Patients with severe learning difficulties

4. Patients with history of failed sedation

5. Patients who may prove difficult to sedate such as alcoholic or drug addicted patients

6. Patients with poor venous access

7. Phobic or uncooperative patients such as children, dementia patients and psychiatric patients

8. Patient being sedated with intravenous propofol

Emergency cases- with high risk of aspiration and requiring endotracheal tube with general anesthesia include:

1. Patients with depressed levels of consciousness

2. Patients associated with encephalopathy

3. Patients suspected bleeding varices 
4. Patients with severe cardiac and/or pulmonary abnormalities

5. Patients unlikely to cooperate during endoscopy procedure

\section{Postprocedural period}

Most of cardiorespiratory adverse events occur during the GIE procedure. Standard monitoring including non-invasive blood pressure, heart rate, pulse oxymetry and electrocardiogram is also routinely used in the postprocedural period. Postprocedural nausea/vomiting and pain need to be rescued especially in the ambulatory patients. Fortunately, a lower incidence of procedural nausea/vomiting and pain after the GIE procedure is observed even in the therapeutic endoscopy [27]. Opioid and cyclo-oxygenase-2-inhibitor can be safely and effectively used for procedural pain in the GIE patients [28].

My previous study showed that periodic objective evaluation of home-readiness revealed that the majority of patients would achieve a satisfactory score on or before 1 hour after the GIE procedure [29]. So that, the patients underwent GIE procedures should be admitted in the recovery room unit at least 30-60 min before discharge. The time to home-readiness by objective evaluation correlated with the type of procedure. Most delay after satisfactory home-readiness scores were reached, were due to non-medical reasons.

Sedation-related cardiorespiratory complications also occur immediately after the GIE procedure. The types of complications in the postprocedural period are similar as in the intraprocedural period. The patients who receive benzodiazepine and/or opioid antagonists should to be closely observed in the recovery room unit longer than the other patients.

\section{Summary}

Although the serious adverse events are rare for the GIE procedural sedation. However, the cardiorespiratory-related complications are common. These complications may be severe if the physicians do not detect and treat the patients earlier. An adequate preprocedural history should be obtained and physical examination performed on all patients. Particular attention should be paid to the patient's physical status and cardiorespiratory system. Appropriate preprocedural assessment and optimization of the patients undergoing moderate or deep sedation are essential to minimize complications. Periodical assessment of the level of sedation and continuous monitoring of cardiovascular and respiratory systems provides timely information. Pulse oxymetry and oxygen supplementation are recommended for the reduction of hypoxemia. Capnography monitoring is considered in the patients undergoing prolonged endoscopic procedures who are at risk of deep sedation. Additionally, the standardized discharge criteria should be used to determine the patient's readiness for discharge. Lastly, the physicians should remember that the risk for un-intended deeper level of sedation may be more common after the stimulation of the endoscopic procedure has been removed. 


\section{Author details}

Somchai Amornyotin*

Department of Anesthesiology and Siriraj GI Endoscopy Center, Faculty of Medicine Siriraj Hospital, Mahidol University, Bangkok, Thailand

\section{References}

[1] Ross C, Frishman WH, Peterson SJ, Lebovics E. Cardiovascular considerations in patients undergoing gastrointestinal endoscopy. Cardiology in Review 2008; 16(2): 76-81.

[2] Romagnuolo J, Cotton PB, Eisen G, Vargo J, Petersen BT. Identifying and reporting risk factors for adverse events in endoscopy. Part I: cardiopulmonary events. Gastrointestinal Endoscopy 2011; 73(3): 579-585.

[3] Vargo JJ. Risks of sedation and analgesia. Techniques in Gastrointestinal Endoscopy 2007; 9: 218-224.

[4] Sharma VK, Nguyen CC, Crowell MD, et al. A national study of cardiopulmonary unplanned events after GI endoscopy. Gastrointestinal Endoscopy 2007; 66(1): 27-34.

[5] Becker DE, Haas DA. Recognition and management of complications during moderate and deep sedation. Part 2: Cardiovascular considerations. Anesthesia Progress 2011; 58(3): 126-138.

[6] British Society of Gastroenterology. Guidelines in Gastroenterology: Complications of gastrointestinal endoscopy. http://www.bsg.org.uk/pdf_word_docs/complications.pdf

[7] Amornyotin S, Chalayonnawin W, Kongphlay S. Propofol-based sedation does not increase rate of complication during percutaneous endosopic gastrostomy procedure. Gastroenterology Research and Practice 2011; Article ID 134819; 6 pages, doi: 10.1155/2011/134819.

[8] Amornyotin S, Kachintorn U, Chalayonnawin W, Kongphlay S. Propofol-based deep sedation for endoscopic retrograde cholangiopancreatography procedure in sick elderly patients in a developing country. Therapeutics and Clinical Risk Management 2011; 7: 251-255.

[9] Amornyotin S, Kachintorn U, Kongphlay S. Anesthetic management for small bowel enteroscopy in a World Gastroenterology Organizing Endoscopy Training Center. World Journal of Gastrointestinal Endoscopy 2012; 4(5): 189-193.

[10] Amornyotin S, Leelakusolvong S, Chalayonnawin W, Kongphlay S. Age-dependent safety analysis of propofol-based deep sedation for ERCP and EUS procedures at an 
Endoscopy Training Center in a developing country. Clinical and Experimental Gastroenterology 2012; 5: 123-128.

[11] Amornyotin S, Prakanrattana U, Chalayonnavin W, Kongphlay S, Kachintorn U. Propofol based sedation does not increase perforation rate during colonoscopic procedure. Gastroenterology Insights 2010; 2(e4): 13-16.

[12] Riphaus A, Stergiou N, Wehrmann T. Sedation with propofol for routine ERCP in highrisk octogenarians: a randomized, controlled study. American Journal of Gastroenterology 2005; 100(9): 1957-1963.

[13] Amornyotin S, Kongphlay S. Esophagogastroduodenoscopy procedure in sick pediatric patients: a comparison between deep sedation and general anesthesia technique. Journal of Anesthesia and Clinical Research 2012; 3: 185. doi: 10.4712/2155-6148.1000185.

[14] Casabianca AB, Becker DE. Cardiovascular monitoring: physiological and technical considerations. Anesthesia Progress 2009; 56(1): 53-61.

[15] Lacy CR, Contrada RJ, Robbins ML, et al. Coronary vasoconstriction induced by mental stress (simulated public speaking). American Journal of Cardiology 1995; 75(7): 503-505.

[16] Pino RM. The nature of anesthesia and procedural sedation outside of the operating room. Current Opinion in Anesthesiology 2007; 20(4): 347-351.

[17] Cacho G, Pérez-Calle JL, Barbado A, et al. Capnography is superior to pulse oximetry for the detection of respiratory depression during colonoscopy. Revista Espanola de Enfermedades Digestivas 2010; 102(2): 86-89.

[18] Becker DE, Haas DA. Recognition and management of complications during moderate and deep sedation. Part 1: Respiratory considerations. Anesthesia Progress 2011; 58(2): 82-92.

[19] Cote GA, Hovis RM, Ansstas MA, et al. Incidence of sedation-related complications with propofol use during advanced endoscopic procedure. Clinical Gastroenterology and Hepatology 2010; 8(2): 137-142.

[20] Qadeer MA, Lopez AR, Dumot JA, Vargo JJ. Hypoxemia during moderate sedation for gastrointestinal endoscopy: causes and associations. Digestion 2011; 84(1): 37-45.

[21] Long Y, Liu HH, Yu C, et al. Pre-existing diseases of patients increase susceptibility to hypoxemia during gastrointestinal endoscopy. PLoS ONE 7(5): e37614, doi:10.1371/ journal.pone.0037614.

[22] Hsieh TC, Wu YC, Ding HJ, et al. Clinically unrecognized pulmonary aspiration during gastrointestinal endoscopy with sedation: a potential pitfall interfering the performance of 18F-FDG PET for cancer screening. European Journal of Radiology 2011, doi: 10.1016/j.ejrad.2010.10.030. 
[23] Amornyotin S, Srikureja W, Chalayonnavin W, et al. Topical viscous lidocaine solution versus lidocaine spray for pharyngeal anesthesia in unsedated esophagogastroduodenoscopy. Endoscopy 2009; 41(7): 581-586.

[24] Amornyotin S, Lertakayamanee N, Wongyingsinn M, et al. The effectiveness of intravenous sedation in diagnostic upper gastrointestinal endoscopy. Journal of Medical Association of Thailand 2007; 90(2): 301-306.

[25] Amornyotin S, Pranootnarabhal T, Chalayonnavin W, Kongphlay S. Anesthesia for gastrointestinal endoscopy from 2005-2006 in Siriraj Hospital: a prospective study. Thai Journal of Anesthesiology 2007; 33(2): 93-101.

[26] American Society of Anesthesiologists. Practice guidelines for sedation and analgesia by non-anesthesiologists. An update report by the American Society of Anesthesiologists Task Force on sedation and analgesia by non-anesthesiologists. Anesthesiology 2002; 96(4): 1004-1017.

[27] Amornyotin S, Phasurin T, Wongnuch P. Pain score within twenty-four hours postendoscopic retrograde cholangiopancreatography: a comparison between diagnostic and therapeutic procedures. Gastroenterology Insights 2009; 1: e(7): 20-23.

[28] Amornyotin S, Chalayonnawin W, Kongphlay S. A randomized controlled trial of preprocedure administration of parecoxib for therapeutic endoscopic retrograde cholangiopancreatography. Journal of Pain Research 2012; 5: 251-256.

[29] Amornyotin S, Chalayonnawin V, Kongphlay S. Recovery pattern and home-readiness after gastrointestinal endoscopy. Journal of Medical Association of Thailand 2007; 90(11): 2352-2358. 